\title{
Percepción sobre Isigni de la comunidad de Sawa, Waspam, río Coco
}

\author{
Rigoberto Guido Blixly' \\ Catalina Yunkiath Cobam ${ }^{2}$ \\ Serafina Espinoza Blanco ${ }^{3}$
}

\section{Resumen}

C sta investigación describe la percepción sobre Isigni de la comunidad de Sawa. Fue un estudio cualitaEtivo con un diseño de historia de vida donde se suministró una entrevista a curanderos, sukias, líderes comunales, ancianos y ancianas de la comunidad, así como grupos focales a estos mismos actores de la comunidad. Los resultados de la investigación determinan que en la comunidad de Sawa se mantienen viva las prácticas de sanación heredado de sus antepasados, tienen el conocimiento de identificar el mal y resolver el problema de salud que provoca el Isigni. Se concluye que la sanación a esta enfermedad consiste en la aplicación de plantas medicinales, oratorias y otros tipos de rituales de sanación.

Palabras clave: Isigni, Sanación, Plantas Medicinales, Espiritualidad, Comunidad.

\section{Summary}

This research describes the perception the Sawa community has about Isigni. It was a qualitative study based with a life history design that was achieve through the application of interviews and focus groups with healers, sukias, community leaders and community elders. The results of the investigation determine that in the community of Sawa the healing practices they inherited from their ancestors are still alive, they have the knowledge to identify the evil and solve the health problem that is cause by the Isigni. Therefore, the study concludes by stating that the healing of this disease consists in the application of medicinal plants, oratories and other types of healing rituals.

Keywords: Isigni, healing, medicinal plants, spirituality, community.

\section{Introducción}

T a investigación se realizó en aras de evidenciar la otra realidad que persiste en las comunidades indíLgenas sobre el proceso salud-enfermedad y los otros saberes y conocimientos que se mantienen en la población cuyas prácticas restablecen la salud y el equilibrio entre las personas, la familia, la comunidad y el mundo espiritual.

Consideramos pertinente y relevante, ir descifrando cada vez más los problemas de salud de origen sociocultural que de una u otra manera causan el desequilibrio en la salud de la población en las comunidades. En esta ocasión nos enfocamos en las siguientes preguntas: ¿Qué es el Isigni?, ¿Qué es lo que provoca en la salud de las personas? y ¿Cómo se curan las personas afectadas?, ya que es una realidad presente en el municipio y en las comunidades. Sin embargo, en esta ocasión nos centraremos en la comunidad de Sawa, como una unidad seleccionada para los efectos de la investigación.

\footnotetext{
1 Técnico Superior en Enfermería, Enfermero responsable del puesto de salud de Sawa. E-mail: rigobertoguidoblixly@gmail.com.

2 Técnico Superior en Enfermería. Enfermera del centro de salud de Waspam río Coco. E-mail. catalinayunkiathcobam@gmail.com

3 Máster en Salud Intercultural con Mención en Salud Sexual y Reproductiva, Directora IMTRADEC de la Universidad de las Regiones Autónomas de la Costa Caribe Nicaragüense. E-mail: serafinaespinoza@gmail.com
} 


\section{Revisión de literatura}

Los pueblos indígenas miskitus de la Costa Caribe Norte mantienen sus costumbres, creencias y prácticas bastante arraigadas a pesar del paso de los tiempos y la culturización que de acuerdo a algunos registros históricos existentes iniciaron en el año 1847 con la llegada de los primeros misioneros. De acuerdo a Figueroa (2004), los miskitus son un pueblo con amplios y profundos conocimientos, y el tema de la muerte no queda exento a estos. Es considerado uno de los aspectos de ritualismo de mayor relevancia en el seno familiar y comunal, y se expresa por sentimientos profundos de mucho dolor, llantos y recuerdos. Esto puede durar varios años incluso perdurar en el tiempo.

Isigni es inmortal, si no se siguen los rituales pertinentes, este puede llegar a convertirse en lasa (demonio o espíritu maligno) y provocar males en la comunidad. Los curanderos de la comunidad de Sawa, afirman que Isigni puede provocar desequilibrio en la salud de las personas de cualquier sexo y edad, sin embargo, es más frecuente que afecte a niñas y niños. Por lo general, la persona afectada es un familiar, pariente o amigo de una persona que ha fallecido sea reciente o haber transcurrido algunos meses $u$ años. Los curanderos refieren que a lo largo de muchos años y desde tiempos muy remotos sus ancestros en su comunidad de Sawa practicaban la medicina tradicional con resultados efectivos, debido a esto, hasta nuestros días es bien respetada, reconocida utilizada por la población.

La relación que tienen los miskitus con el mundo espiritual, es una realidad lo que se constata con la siguiente percepción de uno de los actores claves que participo en la tesis del maestro Figueroa (2004), describe: los miskitus en su agonía ven venir personas desconocidas y extrañas en representación de Dawan (Dios), y también ven llegar a sus familiares cercanos que ya han fallecido, de acuerdo a las creencias este proceso se da para acompañar a los que fallecen en el largo camino hacia el otro mundo donde será su morada eterna. De igual manera, Pérez-Chiriboga (2002), describe lo siguiente:

Los miskitus perciben la vida espiritual propia de los cuatro espacios del ambiente como una fuente de poder superior a la condición humana. El concepto de poder espiritual puede describirse mediante una analogía con el concepto occidental de energía. Es una fuerza o poder latente que existe en el universo, independientemente de los seres humanos, quienes a su vez están bajo la influencia de su presencia ubicua. Esta fuerza carece de propiedades inherentes positivas o negativas, buenas o malas.

Isigni es el espíritu de los muertos y puede causar desequilibrios en la salud de personas adultas, jóvenes y niños; sin embargo, los efectos son más constantes y causa mayor afectación en las niñas y niños menores de cinco años, en algunas ocasiones incluso causar la muerte en menos de 24 horas.

La sabiduría de los sikakaira nani (sabios y sabias de los conocimientos ancestrales) están evidenciadas en varias investigaciones que se han realizado desde la Universidad de las Regiones Autónomas de la Costa Caribe Nicaragüense (URACCAN), a través del Instituto de Medicina Tradicional y Desarrollo Comunitario (IMTRADEC), en dos décadas, deja en evidencia los benéficos que obtiene la población a través de la utilización de la medicina tradicional, la cual en muchas ocasiones se convierte prácticamente en la única opción de sanación y sobrevivencia principalmente para aquellas comunidades que geográficamente se localizan alejadas de las unidades de salud. Entre los estudios de revitalación está práctica novenaria que consiste en rendir honores por última vez a una persona que fallece, se reúnen la familia y las amistades más cercanas. Este proceso es acompañado por reflexiones bíblicas, rezos y cantos. Es un espacio para acompañar a la familia.

\section{Materiales y método}

Este proceso investigativo conllevó un trabajo a nivel de la comunidad de Sawa, con la participación de curanderos, sukias, líderes comunales, mujeres ancianos y ancianas de la comunidad, quienes aún guardan sus conocimientos tradicionales ancestrales. Con el objeto de profundizar en el proceso investigativo se aplicó un enfoque metodológico cualitativo sustentado en diseño de Historia de Vida, porque tratamos de describir los relatos y acontecimientos de las personas con la finalidad de saber la percepción sobre Isigni en la comunidad de Sawa.

Se diseñó una entrevista y una interacción con grupo focal para el cumplimiento de los objetivos planteados. Previo trabajo de campo se realizó 
coordinación, con cada uno de los involucrados en este proceso. El proceso de recopilación de la información se realizó en la lengua materna indígena miskitu.

\section{Resultados y discusión}

\section{Conocimiento que tiene la comunidad sobre el Isigni}

Todos los entrevistados mencionaron que el Isigni es un problema que afecta la salud de las personas desde épocas remotas en la comunidad de Sawa. Puede afectar a adultos y niños, especialmente a los menores de 5 años. Si los pacientes no son atendidos a tiempo pueden presentarse mayores daños en la salud y en ocasiones pueden fallecer. También, respondieron que este problema de afectación por Isigni puede aparecer de forma espontánea, bruscamente, y evoluciona rápidamente, o sea que la persona afectada se puede gravar en cuestión de minutos u horas. Sus antepasados lucharon constantemente con este mal. Para ellos, consiste en una situación compleja que no se logra eliminar totalmente.

Esta enfermedad se presenta de manera esporádica, sin embargo, no deja de estar presente en la comunidad, se debe de tomar en cuenta que ha permanecido a través de los tiempos. El enfermo presenta los siguientes problemas: "mihta bara mina nani kauhla takisa, bla dinkisa, suapni dinkisa", en las manos y los pies, se siente una temperatura bastante fría, que va aumentando conforme avanza el mal, conforme transcurre el tiempo también esta misma parte del cuerpo adquiere un color morado, el paciente está somnoliento o puede permanecer dormido por varias horas, no tiene apetito, hay flacidez muscular, su estado es similar a un enfermo en estado de coma, pues no responde ante ningún estímulo físico, la temperatura corporal sigue bajando hasta quedar todo el cuerpo muy helado similar a la de un cadáver, aunque no exista un factor aparente que desencadene este tipo de temperatura.

Este problema de salud es causado por el espíritu de los difuntos. Por lo que la afectación por lo general está centrada en hogares que han tenido familiares que han fallecido, se puede originar también por visitar cementerios, principalmente en algunas horas y por asistir a velorios. El curandero anónimo de la comunidad refiere que:
[...] los estilos de vida antes eran distintos. Por lo general se mantenía alejado a los niños de un entorno relacionado a un velorio. Los niños no podían, ni tenían que acercarse a lugares donde se estaba velando a una persona fallecida, había personas mayores que se hacían cargo del cuido de los niños llevándolos lejos, incluso se creía que una mujer embarazada no tenía que estar cerca del difunto, ya que podía afectar el feto, si se daba el acercamiento por fuerza mayor, la embarazada tenía que ponerse en tratamiento con pukni [...]

Isigni para los miskitus de la comunidad de Sawa, es cuando el alma abandona el cuerpo humano, abandona el mundo material y se convierte en un ser espiritual, que permanece al lado de sus familiares. Cuando una persona fallece, el espíritu en ocasiones causa daño. Los muertos están cerca, con nosotros, no quieren dejar este mundo y permanecen en sus casas con sus familiares, muchos nos protegen, otros no pueden enfermar.

También se da el fenómeno de visitas nocturnas de los Isigni, ya sea para visitar a sus familiares $y$ amistades, en ese acercamiento pueden hacer contacto con alimentos que se dejan en la cocina. Si esto sucede la persona que ingiere de ese alimento se puede enfermar de kukra. Es por eso, que por lo general los adultos recomiendan no dejar comidas para el día siguiente principalmente en un hogar que ha fallecido recientemente una persona. Lo importante es que existe la cura a través de rituales y se pueden obtener resultados positivos de manera rápida.

En este sentido Pérez-Chiriboga (2002) describe que todo contacto entre humanos y espíritus que ocurra sin el control adecuado, resulta en enfermedad. Los miskitus entienden la enfermedad como posesión espiritual: el espíritu entra en el cuerpo de la persona, donde las fuerzas calientes y frías se repelen, resultando en una afección física o en desórdenes emocionales o mentales.

\section{Proceso de sanación de Isigni}

Para el abordaje del proceso de sanación desde una perspectiva basada en los conocimientos tradicionales ancestrales, los comunitarios se abocan a los curanderos y sukias de su comunidad. Ellos tienen la capacidad de sanar al enfermo del Isigni. Para tal fin en primer lugar se aseguran de estar seguros 
que se trata de una afección originada por Isigni, posteriormente inician el proceso de la recolección de yerbas o plantas medicinales que van a utilizar.

La aplicación por lo general consiste en baños, sobados, y pukni (especie de humazón) en los alrededores de la vivienda del enfermo. Para cada uno de los procesos se tiene una hora especifica. Por lo general, el proceso se aplica por una semana; no obstante, en muchas ocasiones los efectos se ven rápidamente al instante de iniciar el tratamiento, es Kiama lijni, (es de rápido efecto). El proceso se debe de completar con lo programado.

Entre las hierbas más utilizadas se encuentran los siguientes: pabula tagni (san diego), culantro (Eryngium foetidum), mihta paip, daim lupia, sinsinia. Estos se machacan y se aplican en forma de sobado. También se pueden quemar algunas hojas dentro de la casa. El concepto de salud para el indígena es la relación del hombre con la naturaleza. La unidad indivisible es naturaleza-hombre. La naturaleza representa la armonía y el equilibrio físico, mental y espiritual. Reconocen, veneran y respetan la naturaleza porque ofrece, da, y resuelve las necesidades elementales de la vida.

\section{Medidas de prevención}

El pueblo miskitu, como toda cultura milenaria, a través de los tiempos, han sobrevivido a diferentes fenómenos, sean estos de origen natural o sobrenatural y siempre han contado con la participación de los Sikakakaira nani (conocedores de la ciencia milenaria, la medicina indígena), quienes tienen profundos conocimientos sobre las enfermedades de origen sociocultural. Además de conocer los distintos procesos de sanación, también están en la capacidad de aplicar medidas preventivas, alejar los espíritus, y dejar un ambiente de bienestar familiar. Sin embargo, la familia debe de cumplir al pie de la letra las indicaciones. Les prohíben consumir algunos alimentos por unos cortos períodos de tiempo. Otro aspecto es la limpia de la vivienda, para el pueblo miskitu, esta es una práctica común; en general, existe un ambiente de fe y confianza hacia los curanderos y sukias.

\section{Historias de vida: Vivencia de una mujer en la comunidad de Sawa}

La experiencia que narra una de las mujeres adultas de la comunidad de Sawa con relación al Isigni, es que en una ocasión ella sufrió esta afec- tación al estar sentada una tarde en el corredor de su casa, de repente le dio malestar general, mareo, escalofrío. Esto se iba acentuando rápidamente según pasaban las horas. En esos momentos estaba presente una de las hijas, la madre le dijo que la lleve donde el sukia de la comunidad, pues estaba segura que a ella la estaba afectando algo sobrenatural.

La hija entonces la llevó al sukia, este inmediatamente atendió a la persona enferma que había llegado en búsqueda de atención, y él realizó una valoración del estado de la persona, aplicando un ritual para diagnosticar, dándose cuenta que la señora enferma había sido afectada por Isigni. El sukia le aplicó un tratamiento basado en plantas medicinales con el que se realizó sobado por todo el cuerpo, también le entrego un preparado con plantas medicinales y otros materiales para que se haga pukni (bajo) para ser aplicado en los alrededores de la vivienda.

\section{Historia vida: La vivencia de un niño en la comunidad de Sawa}

Un día por la noche temprano el niño jugaba en el patio de su casa, después alguien gritó, cuando la madre fue a verlo él niño estaba llorando y temblando. Lo llevó dentro de la casa y al momento fue teniendo fiebre que se elevaba poco a poco hasta tenerla bien alta, los pies y manos estaban bien helados y en estado somnoliento.

La madre lo llevó al curandero, le explicó todo lo que le sucedió al niño. El curandero pidió $C \$ 20.00$ para la playa mana (pago para recoger la plantita), este es un procedimiento que realiza todo curandero, por el ritual del corte de las hojas y las técnicas que se aplican tomando en cuenta los cuatro puntos cardinales. Una vez que obtuvo la planta medicinal procedió a sobar al niño y una que vez que finalizó el infante se quedó profundamente dormido; más o menos en dos horas, se le fue quitando poco a poco lo helado de las manitas, los pies y la fiebre. Posteriormente se despertó, pero estaba aún somnoliento y con debilidad muscular. La madre le ofreció agua y el niño tomo en poca cantidad. La planta medicinal tubo efectos en la salud del niño, ya que rápidamente se le quito la fiebre, a pesar que los otros malestares fueron desapareciendo poco a poco hasta desaparecer en su totalidad. 


\section{Conclusiones}

En la comunidad de Sawa, aún en esta época, la población tiene problemas de salud originados por la enfermedad del Isigni, este desequilibrio de la salud, les ha afectado por muchos años en su comunidad, sus ancestros luchaban contra este problema, realmente no se ha podido controlar de forma definitiva. Esta enfermedad de filiación cultural afecta a todas las personas de todas las edades y sexos, pero en mayor grado a niños menores de cinco años. El afectado puede ponerse grave rápidamente o sea en cuestión de horas.

En la comunidad de Sawa se mantiene viva la creencia, las costumbres y los estilos de vida, la relación con la naturaleza y los espíritus que en ella moran, lo anterior es parte de su cosmovisión indígena. Existen curanderos en la comunidad que tienen amplios conocimientos para curar el Isigni, esto consiste en la aplicación de tratamientos a base de plantas medicinales. Los comunitarios tienen mucha fe en los curanderos y los beneficios de las plantas medicinales. Para la sanación los curanderos además de las plantas medicinales aplican distintos rituales, invocaciones y procesos de limpia (pukni).

Los curanderos indican medidas preventivas que consiste en: la prohibición del consumo de algunos alimentos, les dan amuletos protectores, hacen limpias en las viviendas afectadas (rituales de limpia), curan a toda la familia, esto una vez que ha concluido el tratamiento y los enfermos han sido sanados. En definitiva, la comunidad de Sawa se mantiene viva la cultura ancestral, para ellos Isigni, es una realidad que afecta en la comunidad, tiene su origen en el contacto que se realiza entre la persona fallecida y los vivos, los afectados se pueden gravar rápidamente si no se busca la cura. El tratamiento consiste en la aplicación de plantas medicinales.

\section{Bibliografía}

Asamblea Nacional. (2011). Ley No. 759, Ley de Medicina Tradicional Ancestral.

Millan, A. (2008). Investigación Cualitativa.

Cox, A. (2003). Tulu Walpa.

Figueroa, R. (2004). La Celebración de la Muerte: Un Estudio de la Herencia Cultural en la Iglesia Morava Central Miskita en Bilwi, Nicaragua desde una Perspectiva Pastoral. RACCN: Iglesia Morava

FAPCI. (2005). Primera Conferencia Internacional en el Marco de Fórum de las Culturas de Barcelona. Medicina tradicional indígena, interculturalidad y globalización.

Iku, H. (2005). Cosmovisión de los Pueblos Indígenas.

Pérez-Chiriboga, I. (2002). Espíritus de Vida y Muerte.

Rizo, M. (1999). Los Dimalah deidades o espíritus benévolos que instruían al sukia. 